\title{
Architecture to Integrate Multiple PONs with Long Reach DWDM Backhaul
}

\author{
Darren P Shea and John E Mitchell, Member, IEEE,
}

\begin{abstract}
This paper demonstrates the feasibility of an architecture that consolidates a number of deployed Passive Optical Network (PON) infrastructures into a long-reach, high-split ratio system which further increases equipment sharing between users. The demonstrated system allows the use of uncooled lasers with possible wavelength drift across a CWDM band $(20 \mathrm{~nm})$ with optical amplification and narrow optical filtering with no performance degradation. A complete study of potential implementations was performed with experimental results showing that a target performance of $10^{-10}$ could be achieved over $120 \mathrm{~km}$ of standard fiber with transmitter wavelengths from 1542 to $1558 \mathrm{~nm}$ and DWDM backhaul wavelengths from 1520 to $1535 \mathrm{~nm}$. This gives the potential to support up to 2560 users.
\end{abstract}

Index Terms-PON, Wavelength Conversion, DWDM, XGM

\section{INTRODUCTION}

$\mathbf{P}$ ASSIVE optical networks (PON) are a key technology to enable the development of an optical access network. Currently there are many versions of PON technology [1], [2]. First developed by BT in the 1980s [3] the technology was furthered with the development of the Broadband PON to carry broadband data over $20 \mathrm{~km}$ to 32 customers at symmetrical data rates of up to $622 \mathrm{Mbit} / \mathrm{s}$ [4]. The IEEE has also developed a PON architecture as part of the Ethernet in the First Mile project [5]. Ethernet-PON (EPON) are capable of delivering Ethernet encapsulated data at a rate of $1 \mathrm{Gbit} / \mathrm{s}$ symmetrical with commercially available systems demonstrating 64 users over a range of up to $20 \mathrm{~km}$ [1]. However, the highest capacity standardised PON architecture is the GigabitPON standardised by the ITU-T, which is capable of operating at $2.5 \mathrm{Gbit} / \mathrm{s}$ symmetrical data rates, connecting up to 64

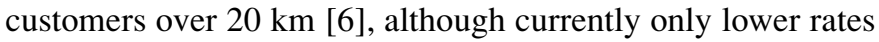
$(1.25 \mathrm{Gbit} / \mathrm{s})$ in the upstream are commercially available. Traditionally PONs are connected to the core telecoms network through SDH rings which form the metro network. Large cost savings could be made by implementing a combined access/metro optical solution as proposed by the ACTS project PLANET [7]. The SuperPON concept was further refined with the development of a Long Reach Optical Access Network (LROAN) [8]. Both systems enable significant cost savings by implementing large split sizes, long-reach, high-speed optical

Manuscript received 15 January 2008; revised 15 August 2008. The work described in this paper was carried out with the support of the BONE-project (Building the Future Optical Network in Europe), a Network of Excellence funded by the European Commission through the 7th ICT-Framework Programme.

D. P. Shea and J. E. Mitchell are with University College London (UCL), Department of Electronic \& Electrical Engineering, Torrington Place, London WC1E 7JE, UK (e-mail: jmitchel@ee.ucl.ac.uk).

Digital Object Identifier 10.1109/JSAC.2009.090204. access networks. PLANET [2], developed in the late 90s, enabled 2048 customers to be connected over $100 \mathrm{~km}$ at $2.5 \mathrm{Gbit} / \mathrm{s}$ downstream and $311 \mathrm{Mbit} / \mathrm{s}$ upstream. LROAN provided similar reach $(110 \mathrm{~km})$ at $10 \mathrm{Gbit} / \mathrm{s}$ symmetrical to 1024 customers.

In developing these systems further there is a requirement that DWDM operation be implemented in the backhaul to increase the fiber efficiency. In the simplest implementation, this would require tunable lasers in the customer equipment with accurate wavelength locking or wavelengths supplied from the central office and modulated at the optical network unit (ONU) by a device such as a reflective SOA. Such techniques have been demonstrated recently in the EU project PIEMAN [2]. However, we suggest that significant savings could be made by using a technique that allows uncooled, wavelength variable sources to be used in the customers ONU while still enabling tight optical filtering to limit amplifier noise and support multiple wavelengths in the backhaul. Using a combination of a generalised local access PON and a wavelength converter, it is possible to create an optical access network which presents all of the advantages of PON, long reach access and DWDM.

In this paper, we experimentally demonstrate a $20 \mathrm{~km}$ Wavelength Converting Optical Access Network (WCOAN) with $100 \mathrm{~km}$ DWDM backhaul and $20 \mathrm{~km}$ PON distribution section. This enables uncooled lasers with wavelength drift across a CWDM band, to be used in a long-reach optical access network by implementing wavelength conversion prior to the DWDM backhaul. Section II describes the network architecture proposed, based on the concept of consolidating multiple existing PON architectures into a single integrated DWDM backhaul, with section III outlining the experimental set-up. Section IV demonstrates an integrated system of two SOA at the wavelength conversion point, and shows the possibility of consolidating PONs with 32 way splits. Section $\mathrm{V}$ extends this proof of concept by considering a system where the gain slope effects of the first SOA stage are reduced and increases the split size to 64 as well as demonstrating operation with two simultaneous channels. In section VI we demonstrate that with the addition of an amplification stage in the backhaul, it is possible to increase the number of ONUs on each PON to 128 , thereby enabling a total of 2560 ONUs to be consolidated onto the 20 DWDM channels available.

\section{ARChitecture}

A major obstacle for the optical access network is the cost of the ONU installed at the customer premises [1]. Current PONs reduce this burden by using low cost uncooled transmitters in the ONU. However, this results in the transmission 


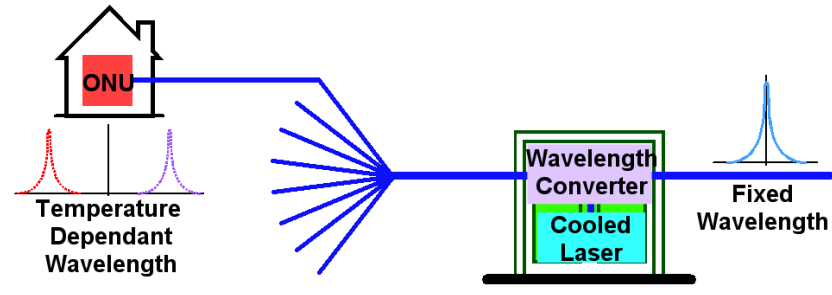

Fig. 1. Conversion between temperature dependant wavelength and a DWDM wavelength.

wavelength being temperature dependant with a possible drift of $\pm 10 \mathrm{~nm}$. As no components in either direction of standard PONs (EPON or GPON) are wavelength critical to less than $\pm 10 \mathrm{~nm}$, the performance is unaffected. The inclusion of wavelength stable sources is currently needed both when multiple wavelengths are used to enable DWDM operation in the backhaul as well as when optical amplification is included to overcome the large amount of additional loss introduced by incorporating a large split and/or long fiber length, where optical filtering is used to reduce the effects of ASE. Hence, in long reach systems, more expensive, cooled transmitters are used to ensure stable wavelengths.

The concept of our proposed system is shown in Figure 1. The inclusion of a wavelength converter means that drift of the transmitter wavelength has no effect on system performance as it is converted to a stable wavelength before the narrow-band filtering in the optical path. Via the wavelength conversion process, the network operator has complete control over the wavelength to which the original wavelength (pump wavelength) is converted.

The conversion to a stable wavelength has a major advantage; a number of PONs can be grouped together over the same backhaul fiber, the data from each PON being converted to a separate wavelength. This will reduce the overall cost per customer as the utilisation and efficiency of the backhaul fiber is increased allowing the cost to be spread over a larger number of customers. Therefore, with the incorporation of a wavelength converter, a high-split, long-reach optical access network combining both access and metro networks can be created from standard PON architectures and the existing metro backhaul, as illustrated in Figure 2.

A number of wavelength conversion techniques are available. The main techniques used are derived from non-linear effects, namely: four wave mixing (FWM) [9], [10], cross phase modulation (XPM) [11] and cross gain modulation (XGM) [11]. The simplest technique for all optical wavelength conversion involves XGM. XGM can be performed by using the pump wavelength (which carries the data) to saturate an SOA along with a counter or co-propagating probe (CW) wavelength. The gain which the probe experiences is modulated by the pump wavelength causing the inverse of the data signal to be imposed onto the probe wavelength [11]. The simplicity of the XGM conversion is countered by a limited wavelength conversion range where the extinction ratio of the pump is maintained after transfer to the probe. Extinction ratio conservation can only be achieved when converting to a probe wavelength which is shorter than the pump wavelength [11]. Therefore, the performance of the system will be reduced if

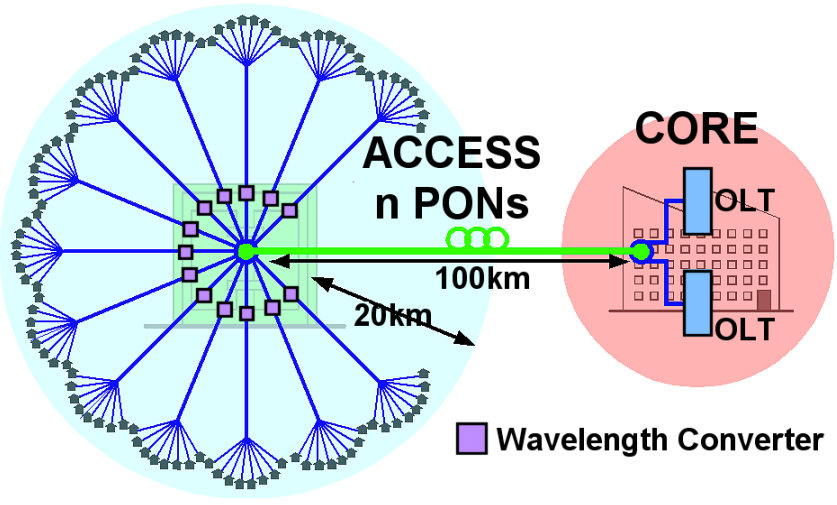

Fig. 2. Formation of long reach high split combined access and metro network from existing standard PONs through the use of wavelength conversion.

the pump is converted to a longer wavelength (e.g. $1550 \mathrm{~nm}$ to $1570 \mathrm{~nm}$ ).

This reduction in extinction ratio can be explained by considering the dependence of SOA saturation power on wavelength due to the gain peak shift due to bandfilling. Increasing the signal power causes the SOA signal gain to saturate due to a reduction in the number of injected carriers. As a result of the decrease in carrier density, the gain peak shifts towards a longer wavelength in accordance with the reverse process of bandfilling [12]. The saturation input power will be lower for a longer wavelength due to the increased gain. Hence, when converting from a shorter to longer wavelength the extinction ratio is reduced as it is more difficult for a shorter wavelength to saturate the SOA. Secondly, the XGM conversion process always results in positive chirp [13], which in turn results in increased pulse broadening during propagation through SSMF. Positive chirp in the converted signal (Probe wavelength) is due to phase modulation, which is caused by changes in refractive index as a direct result of the carrier density modulation [14]. Hence, the propagation distance of the converted signal is limited by the positive chirp.

\section{EXPERIMENTAL ARCHITECTURE}

The experimental, upstream, dual fiber architecture of the long reach WCOAN is shown in Figure 3. The focus was on the upstream transmission as it presents the most difficulties due to the cost sensitivity of the ONU. In the distribution section, a single fiber infrastructure which follows the GPON standard was represented by a $20 \mathrm{~km}$ of fiber $(\mathrm{SSMF}, \alpha=0.25 \mathrm{~dB} / \mathrm{km}$, Dispersion $=16 \mathrm{ps} / \mathrm{nm} . \mathrm{km})$ and an optical attenuator were used to represent the attenuation inherent in the optical splitter. An insertion loss of $3.5 \mathrm{~dB}$ per 2 way split was assumed with the additional $0.5 \mathrm{~dB}$ added to account for non-ideal device construction. For example, the insertion loss equivalent to a 32 way split is $17.5 \mathrm{~dB}$ (32= $2^{5} ; 3.5$ x $5=17.5 \mathrm{~dB}$ ). In the experiment the ONU transmitter consisted of a tunable laser with an external MZ modulator operating at $2.5 \mathrm{Gbit} / \mathrm{s}$ to simulate the possible wavelength drift of an uncooled, directly modulated ONU transmitter which would be mandated in a commercial realization. The transmitter had an output power of $2 \mathrm{dBm}$ with an extinction ratio of $10 \mathrm{~dB}$ which complies with the GPON standard. The 

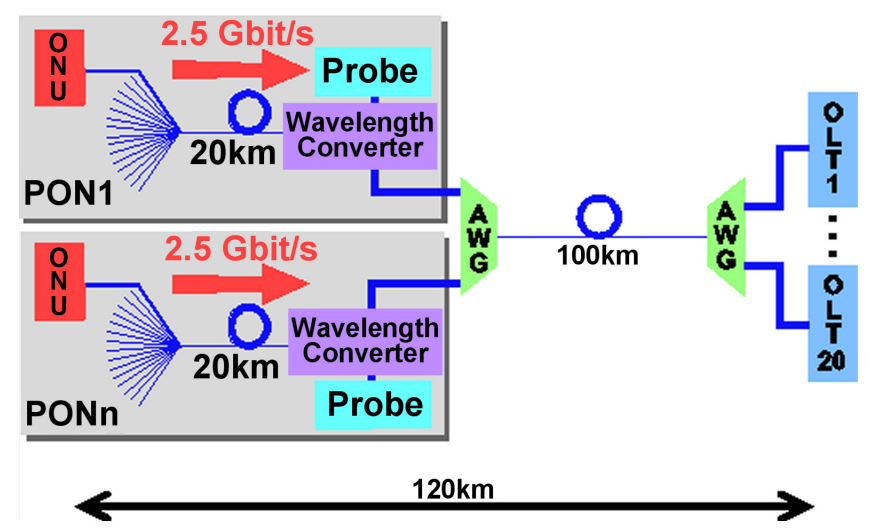

Fig. 3. General experimental architecture of the upstream channel for a WCOAN.

transmitter wavelength was $1550 \mathrm{~nm} \pm 10 \mathrm{~nm}$, following the wavelength plan suggested previously for long reach optical access networks [15]. As in previous systems, a dual fiber backhaul would be used to avoid any wavelength overlaps between the upstream and downstream channels [8]. This may also reuse existing fiber pairs; for example those installed to provide SDH backhaul to current systems. After propagation through the PON distribution section the data signal was transferred to the probe wavelength in the XGM wavelength converter. The probe wavelength was supplied by a CW DWDM laser. Exact details of the wavelength converter will be given in the subsequent sections.

Directly after the wavelength converter was the backhaul section. The converted signal, at the probe wavelength, was wavelength multiplexed into a $100 \mathrm{~km}$ SSMF backhaul through an arrayed wavelength grating (AWG). A second AWG was positioned immediately after the backhaul fiber to filter each channel to the correct Optical Line Terminal (OLT). The OLT was formed of a $2.5 \mathrm{Gbit} / \mathrm{s}$ avalanche photodiode (APD) receiver with sensitivity of $-31 \mathrm{dBm}$ at $\mathrm{BER}=10^{-9}$. The AWGs had 20 channels with $100 \mathrm{GHz}$ spacing, allowing DWDM to be implemented in the backhaul.

\section{INITIAL SYSTEM EXPERIMENT}

The first implementation used two SOAs in the wavelength conversion unit: one acting as a pre-amplifier to boost the power of the incoming signal to a point where it was possible to saturate the second SOA, which performed the conversion. The use of two SOAs is ideal as it creates a device that is tolerant to bursty data and that could be integrated, if required.

\section{A. Experimental Setup}

The first experimental setup to be analysed used the general architecture presented in Figure 3. As the XGM wavelength conversion process relies on SOA gain saturation, an SOA preamplifier stage was required to ensure there was sufficient optical signal power to saturate the SOA acting as the wavelength converter, as depicted in Figure 4. To improve the performance of the XGM wavelength conversion process, a CWDM optical filter ( $20 \mathrm{~nm}, \lambda=1550 \mathrm{~nm}$ ) was used to reduce SOA saturation through amplified spontaneous emission (ASE). The probe wavelength $(1533.47 \mathrm{~nm})$, which the signal was converted

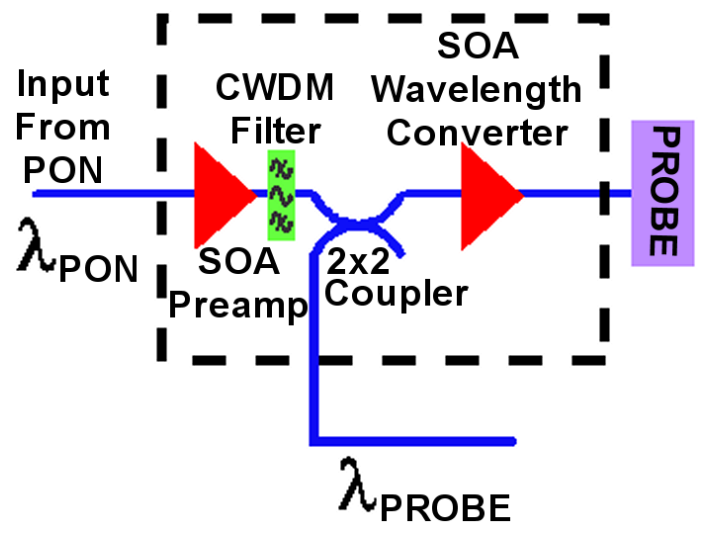

Fig. 4. WCOAN performance against split size. Performance better than $\mathrm{BER}=10^{-10}$ can be achieved with a split size of 32 .

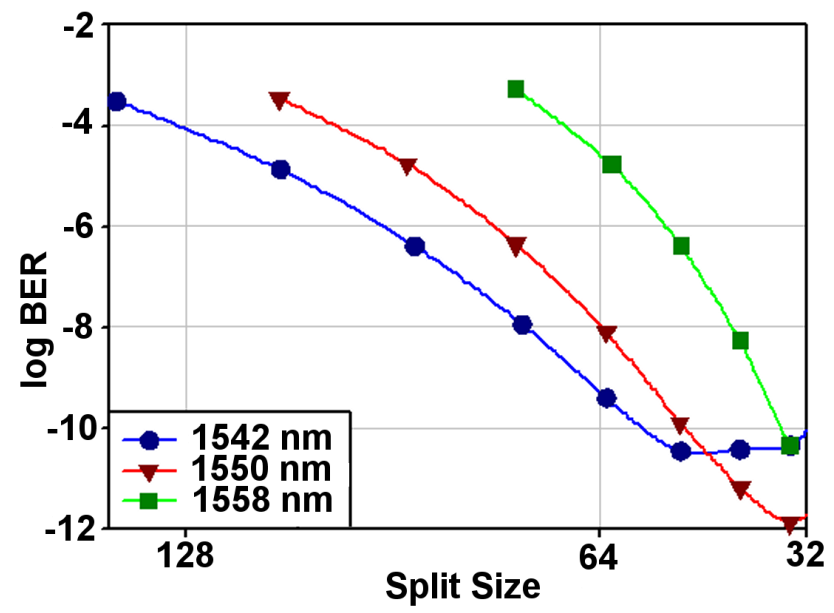

Fig. 5. Wavelength converter for the 32 way split system using an SOA pre-amplifier and an SOA to provide the XGM wavelength conversion to the counter propagating probe wavelength.

to, was supplied by a CW laser, injected into the wavelength converter in the counter propagating direction relative to the data signal (pump wavelength). The signal on the converted wavelength was then coupled out of the wavelength converter through a $2 \times 2$ coupler.

\section{B. Experimental Results}

To assess the performance, a number of experimental tests were carried out. For each test, the optical carrier was modulated with a continuous, NRZ, $2^{23-1}$ PRBS with the optical attenuator set to simulate the splitter loss. Figure 5 shows the BER performance for each wavelength, as the split size increases. The system performance was then measured by varying the split size through adjustments of the variable attenuator, which simulates the splitter in each PON.

The results presented in Figure 5 confirmed that it was possible to achieve the GPON target performance of BER $=10^{-10}$ for individual 32 way splits over $120 \mathrm{~km}$, using 20 channel AWGs. At the input to the XGM wavelength converter, after the distribution section consisting of an optical splitter and $20 \mathrm{~km}$ of SSMF, the $2 \mathrm{dBm}$ ONU transmitter power was attenuated to $-20.5 \mathrm{dBm}$. To ensure that the best possible performance was achieved for all wavelengths, the optimum 


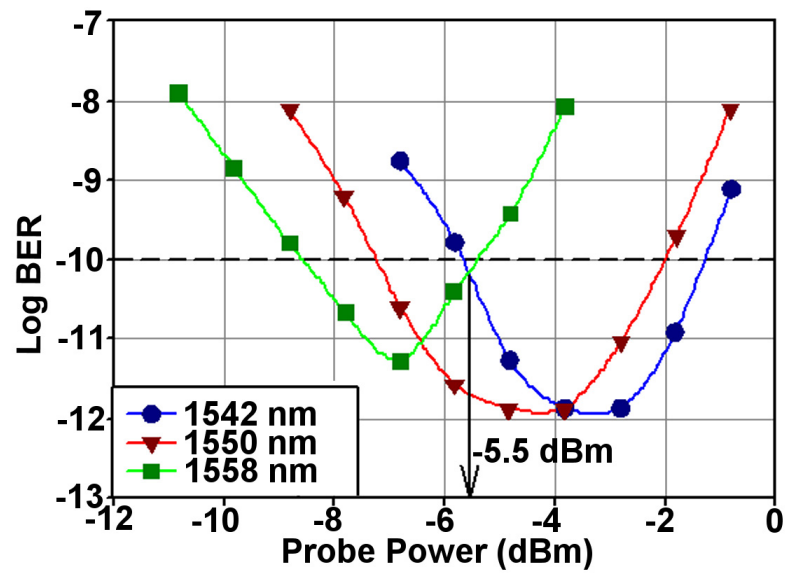

Fig. 6. Probe power optimisation for each wavelength (1542, 1550 and 1558 $\mathrm{nm})$.

probe power was measured. The tunable source, $(\mathrm{P}=2 \mathrm{dBm}$, $\mathrm{ER}=10 \mathrm{~dB})$ was tuned to three wavelengths $(1542,1550$ and $1558 \mathrm{~nm}$ ) to represent possible wavelength drift of the ONU transmitter across a CWDM band centered at $1550 \mathrm{~nm}$. Based on these results a maximum split size of 32 was found. For each wavelength, the optimum probe power was determined by measuring the system performance as the probe power was varied. Figure 6 shows that the optimum is different for all three wavelengths. A probe power of $P_{\text {Probe }}=-5.5 \mathrm{dBm}$ was chosen as the optimum as it will allow each wavelength in the CWDM band to operate with a performance better than $\mathrm{BER}=10^{-10}$.

\section{Discussion}

In all of the experimental results, it was clear that the performance of the $1558 \mathrm{~nm}$ signal was worse than the other wavelengths due to the wavelength dependency of the SOAs used in the experiment. As the input power to the pre-amplifier SOA was $-20.5 \mathrm{dBm}$ for all wavelengths, the pre-amplifier operated in its linear region. The gain peak of the SOA was situated at $\sim 1480 \mathrm{~nm}$, which resulted in a lower gain at $1558 \mathrm{~nm}$ than $1542 \mathrm{~nm}$ due to the negative gradient of the SOA gain profile. At $1558 \mathrm{~nm}$, the signal present at the input of the wavelength converting SOA was $2.7 \mathrm{~dB}$ less than the $1542 \mathrm{~nm}$ signal. A lower input power to the wavelength converter resulted in a less efficient conversion due to the dependence of the XGM process on saturating the second SOA [11]. Due to bandfilling effects, the gain peak of the SOA was shifted to a higher wavelength which increased the saturation power of the SOA at longer wavelengths [12]. Therefore, a higher input power was required at $1558 \mathrm{~nm}$ to saturate the SOA, which reduced the quality of the XGM conversion resulting in a reduced performance. Hence, the reason for reduced performance at $1558 \mathrm{~nm}$ is twofold; reduced gain in the pre-amplifier SOA and increased saturation power in the wavelength conversion SOA. However, it must be noted that the GPON target performance of $B E R=10^{-10}$ can still be achieved.

\section{System Operation with inCREASEd Gain Flatness}

To investigate the potential performance with variable and flattened gain the SOA pre-amplifier was replaced with an EDFA which had a flatter gain profile in the $1550 \mathrm{~nm}$ wavelength region. The deficiencies in system performance due to low gain at $1558 \mathrm{~nm}$ were significantly reduced due to a much stronger signal present at the input to the XGM wavelength converter e.g. at $1558 \mathrm{~nm} P_{O U T_{-} E D F A}=7.1 \mathrm{dBm}$ and POUT_SOA $=-1.44 \mathrm{dBm}$. This resulted in an improved wavelength conversion, as illustrated by the eye diagrams of the converted signals at the output of the wavelength converter shown in Figure 7. The extinction ratios were $5.13 \mathrm{~dB}$ and $6.56 \mathrm{~dB}$ for the SOA and EDFA respectively.

\section{A. Results and Discussion}

Figure 8 shows the performance when two channels were operated simultaneously. Each channel consisted of an individual PON with a XGM wavelength converter in the distribution section and a common DWDM backhaul. For each channel, the ONU wavelength was fixed at $1550 \mathrm{~nm}$ and modulated with a continuous, NRZ, $2^{31-1}$ PBRS. The probe wavelengths were $1531.90 \mathrm{~nm}$ and $1533.47 \mathrm{~nm}$ for PON 1 and PON 2 respectively. Each probe power was optimized individually to enable each wavelength converter to operate with the lowest possible pump signal power. The optimum probe powers were $-0.9 \mathrm{dBm}$ and $-2.7 \mathrm{dBm}$ for PON 1 and PON 2 respectively, which can be attributed to differences in the EDFA preamplifiers used in each PON. The performance of each PON was analyzed by varying the split size through adjustments of the variable attenuator which simulated the split in each PON. As the split reduces, the input power to the SOA increases, driving the SOA beyond the optimum saturation which degrades the converted signal [11]. Optimization of the probe power would allow the network operator to control the performance from the central office, with a resolution of the order of only a $\mathrm{dB}$ necessary to given improved performance. It was possible to achieve performance of $\mathrm{BER}=2 \times 10^{-11}$, in excess of the GPON target of $\mathrm{BER}=10^{-10}$, for individual 64 way splits over $120 \mathrm{~km}$, using 20 channel AWGs. It is important to note that the system performance was measured without the use of forward error correction (FEC). If ReedSolomon $(255,239)$ FEC were to be used, as specified in the GPON standards, the pre-FEC performance BER for target performance $\left(\mathrm{BER}=10^{-10}\right)$ would be reduced to BER $=2.9 \times 10^{-4}[15]$. This would allow the individual GPON split sizes to be increased to 128 . Hence, the total system capacity would be potentially 2560 ONUs.

The ability of the WCOAN to cope with wavelength drift of the source laser in the ONU was demonstrated using the same procedure as before, giving the results shown in Figure 9. A penalty of $0.8 \mathrm{~dB}$ exists between $1542 \mathrm{~nm}$ and $1550 \mathrm{~nm}$ with a larger penalty of $1.2 \mathrm{~dB}$ between $1550 \mathrm{~nm}$ and $1558 \mathrm{~nm}$. Although the gain profile of the EDFA was flatter than that of the SOA some penalties still remain due to the gain slope. At $1542 \mathrm{~nm}$, the output power of the EDFA was $3.25 \mathrm{~dB}$ greater than the output power at $1558 \mathrm{~nm}$. This resulted in better XGM wavelength conversion at $1542 \mathrm{~nm}$ due to deeper gain saturation in the SOA. However, the 
SOA Pre-Amplifier

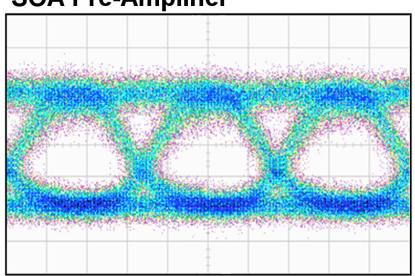

EDFA Pre-Amplifier

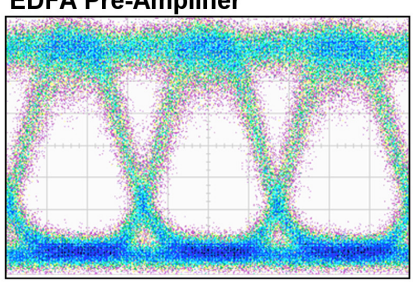

Fig. 7. Comparison of eye diagrams for the converted signal after the wavelength converter when using an SOA and EDFA pre-amplifier. Both eyes are recorded on a $50 \mu \mathrm{V} /$ div scale.

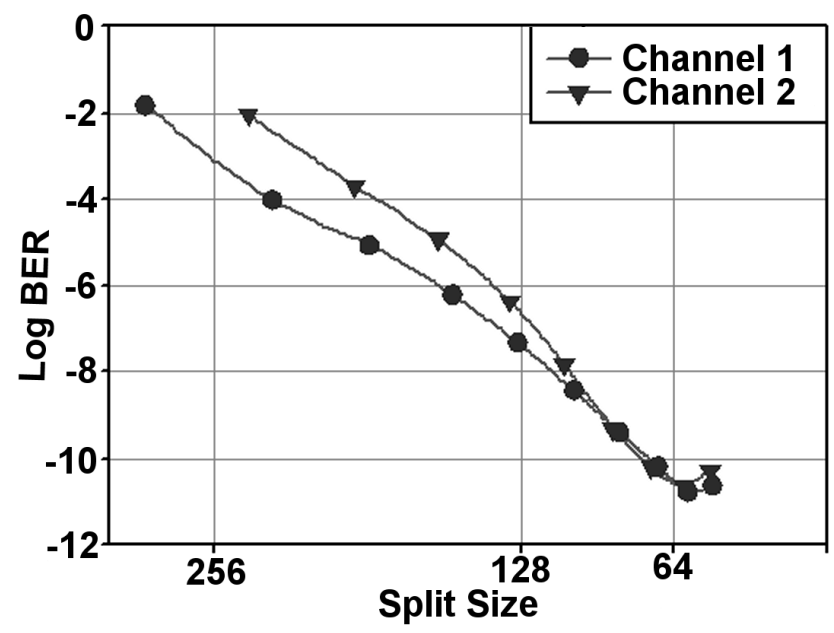

Fig. 8. Two channels operating simultaneously across WCOAN. Both channels achieve better than $\mathrm{BER}=10^{-10}$ with individual 64 way splits giving a potential split of 1280 or 2560 with FEC.

wavelength dependency of the SOA reduces the penalty. At $1558 \mathrm{~nm}$, a higher output power $(6.6 \mathrm{dBm})$ was possible than at $1542 \mathrm{~nm}(4.2 \mathrm{dBm})$, due to increased SOA saturation powers at longer wavelengths. The penalty between wavelengths was therefore reduced as a greater optical signal power was present at the receiver; $-25.7 \mathrm{dBm} @ 1558 \mathrm{~nm}$ compared with-28.1dBm@1542nm.

\section{128 Split With AMPLIFIEd BackHaUl}

Up to this point, all of the system improvements have been made through modifications to the wavelength converter. The network backhaul is another area where performance enhancements can be achieved. After the wavelength converter the signal was subject to $35 \mathrm{~dB}$ of attenuation in the backhaul section due to fiber loss $(25 \mathrm{~dB})$ and insertion losses in the AWGs ( $5 \mathrm{~dB}$ each). An avalanche photodiode (APD) was used in the OLT to recover the optical signal. Further improvements in the receiver sensitivity were made by using an optical amplifier to overcome the loss in the backhaul and AWG. Typically, the benefits of the avalanche gain within an APD are negated when pre-amplified by an optical amplifier. However, here it is placed before the AWG to provide gain as the system is currently limited by noise from the photodiode due to the long backhaul and AWG loss; improving the sensitivity of the receiver and hence the system performance.

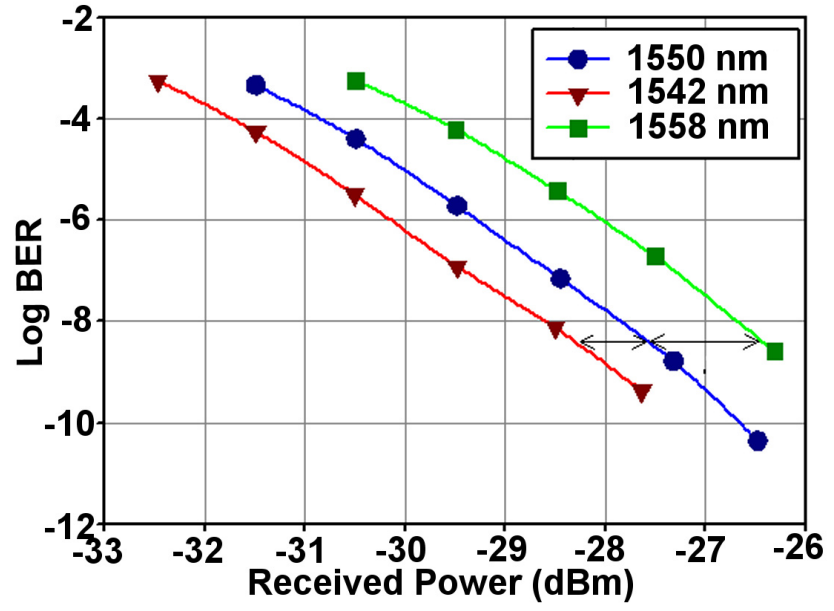

Fig. 9. Wavelength drift resilience of the WCOAN. A tunable source was used to simulate wavelength drift in an uncooled ONU laser. A $2 \mathrm{~dB}$ penalty was observed between $1542 \mathrm{~nm}$ and $1558 \mathrm{~nm}$.
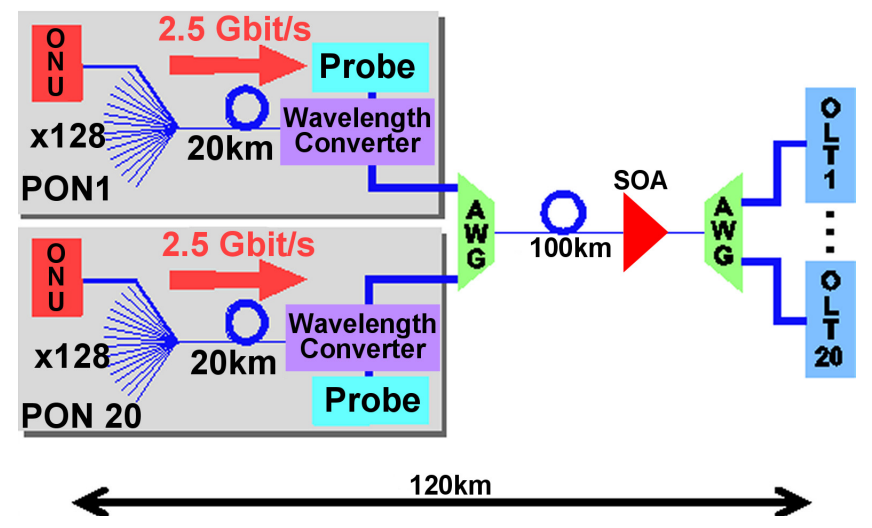

Fig. 10. 128 way split architecture with optically pre-amplified receiver.

\section{A. Experimental Setup}

The modified system architecture is depicted by Figure 10 , where an SOA is placed after the backhaul fiber. In this configuration the second AWG will also act as an ASE filter at the receiver. In this particular setup the wavelength converter uses an SOA pre-amplifier as in the system shown in Figure 4.

\section{B. Experimental Results}

The experimental procedure used to determine whether the performance of the system with amplified backhaul was the same as in the previous sections. Initially, the optimum probe power was determined to be $-26 \mathrm{dBm}$ when using a backhaul wavelength of $1530.04 \mathrm{~nm}$. Figure 11 shows how the optimum probe power enabled the system to operate with a performance better than BER $=10^{-10}$ for a split ratio of 128 across a CWDM band centred at $1550 \mathrm{~nm}$. The performance variation with wavelength corresponds to the wavelength dependency of the SOA identified in Section IV. An increase to 256 way split PONs would be possible when using an EDFA pre-amplifier in the wavelength converter. However, the benefit of using two SOAs is that both devices can be integrated into the same package which creates a much more compact solution. 


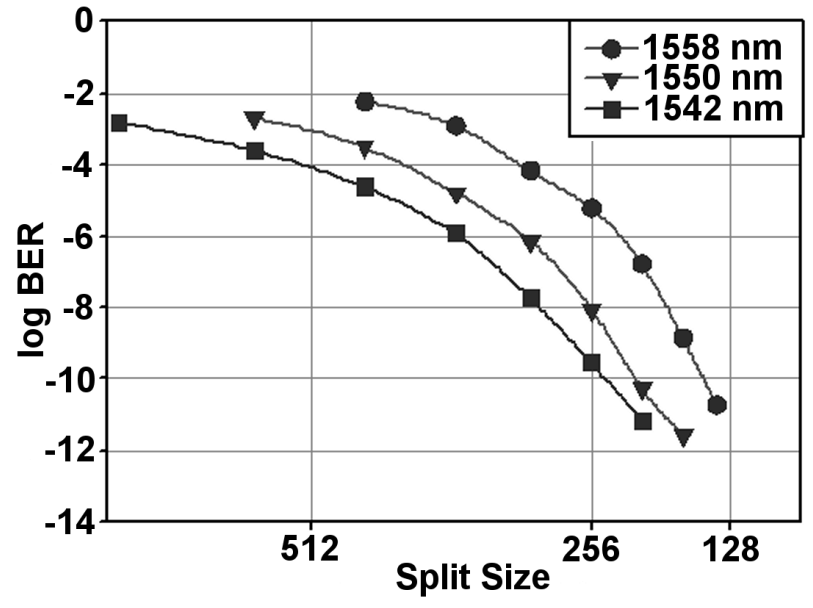

Fig. 11. Performance of the amplified backhaul system showing that a split size of 128 is achievable when the ONU wavelength varies over CWDM channel centred at $1550 \mathrm{~nm}$.

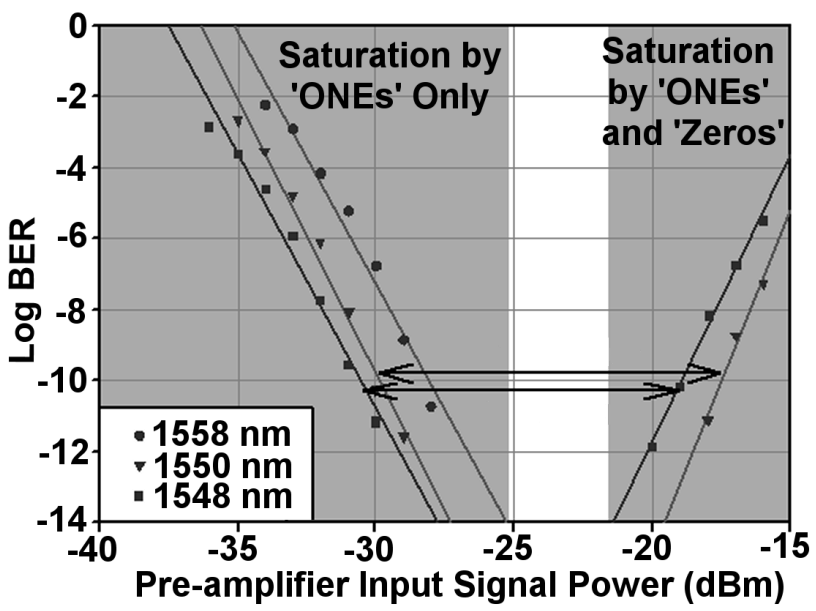

Fig. 12. Dynamic range of the network measured for distribution wavelengths which span a CWDM channel. Shaded areas indicate where the wavelength converter is saturated by ones only (left) and ones and zeros (right).

The dynamic range of the system is shown in Figure 12. The dynamic range of the system defines the range of pump signal power over which the target performance of $B E R=10^{-10}$ can be maintained. Out of the three wavelengths used to simulate the CWDM band centred on $1550 \mathrm{~nm}$, the minimum dynamic range of $11.6 \mathrm{~dB}$ was achieved for $1542 \mathrm{~nm}$ wavelength. Due to the wavelength dependency of the SOAs the longer wavelengths have less gain. This enables the shorter $1542 \mathrm{~nm}$ wavelength to operate with a larger split size. However, higher gain means that the zero level signal will begin to saturate the wavelength converter at lower input power. Saturation of the SOA by the zeros would result in a reduced extinction ratio in the converted signal and hence reduced performance. For longer wavelengths, lower gain means that zero saturation of the SOA is limited, which leads to a larger dynamic range. At $1558 \mathrm{~nm}$, it was not possible to measure the dynamic range due to power limitations i.e. it was not possible to achieve the signal power required for the zero level SOA saturation, indicating that it was in excess of $15 \mathrm{~dB}$.

In a deployed system, numerous DWDM probe wavelengths would be used to carry the data from numerous PON distri-

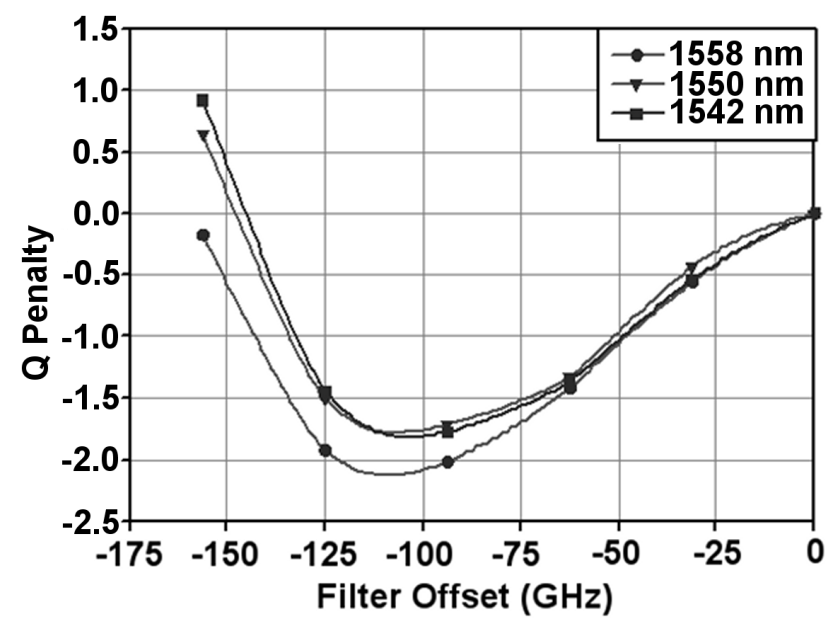

Fig. 13. Performance penalty measure in terms of the $\mathrm{Q}$ factor for various degrees of detuning of the optical filter prior to the receiver.

bution sections connected to the common backhaul through wavelength converters. To ensure that the target BER of $10^{-10}$, can be achieved at different backhaul wavelengths, the system performance was measured at using probe wavelengths of $1520.25 \mathrm{~nm}$ and $1535.04 \mathrm{~nm}$ which provided $20,100 \mathrm{GHz}$ spaced channels. Modifications were made to the experimental set-up to allow tunability of the backhaul wavelengths i.e. the AWGs were replaced with $100 \mathrm{GHz}$ optical filters. Additional attenuation of $35 \mathrm{~dB}$ was included to ensure that the total backhaul attenuation was consistent with the previous experimental setup. It was assumed that the best performance would be achieved when the optical filter centre frequency was perfectly aligned with the backhaul wavelength, as the maximum optical signal power would be present at the receiver. However, this was not the case. Figure 13 shows that the best system performance occurs when the optical filter prior to the receiver is detuned from the centre wavelength by $-110 \mathrm{GHz} \pm 5 \mathrm{GHz}$ $(-0.9 \mathrm{~nm} \pm 0.04 \mathrm{~nm})$.

The reason for this can be explained as follows: driving an SOA into saturation induces phase modulation due to carrier density changes [16]. In the XGM wavelength conversion process different levels of saturation are achieved for ones and zeros in order to modulate the gain of the SOA. This results in a frequency difference between the ones and zeros in the signal frequency spectrum. By detuning the optical filter, it is possible to use the filter to remove the zero component of the signal [17]. Hence, the extinction ratio of the signal is increased resulting in a performance increase. To take advantage of the performance increase the optical filters in the subsequent results are detuned by $-110 \mathrm{GHz}$.

As in the previous sections, wavelength drift of the ONU transmitter across a CWDM band centred at $1550 \mathrm{~nm}$, was simulated by using three test wavelengths, 1542, 1550 and $1558 \mathrm{~nm}$. An attenuator placed prior to the receiver was used to adjust the received optical power in $1 \mathrm{~dB}$ steps. The optimum probe powers were determined to be $-9 \mathrm{dBm}$ and $-7.5 \mathrm{dBm}$ for the $1535.04 \mathrm{~nm}$ and $1520.25 \mathrm{~nm}$ probe wavelengths respectively. The difference between the two optimum probe powers can be attributed to the wavelength dependency of the SOA devices used. The peak gain of the 


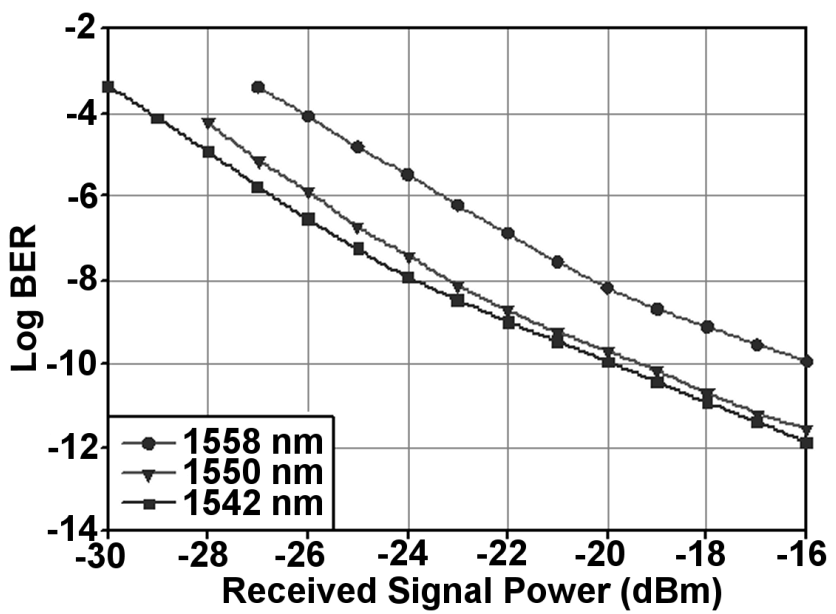

Fig. 14. System performance against received power when converting the distribution wavelengths to a backhaul wavelength of $1535.04 \mathrm{~nm}$.

SOA devices used was at $1480 \mathrm{~nm}$. Hence, the small signal gain of the device increased as the wavelength decreases. Therefore, at $1520.25 \mathrm{~nm}$ the device had a higher gain and required more power from the pump/probe combination so that the device could be saturated ensuring successful wavelength conversion through the XGM process.

The main conclusion taken from the system performance results at the two extremes of backhaul wavelengths, given in Figure 14 and Figure 15 was that the target BER of $10^{-10}$ was achievable with wavelength drift across a CWDM band across backhaul wavelengths from $1520.25 \mathrm{~nm}$ to $1535.04 \mathrm{~nm}$. Subtle differences between the system performance at the ONU test wavelengths for each backhaul wavelength do exist. These differences can be attributed to the choice of probe wavelength. For the $1535.04 \mathrm{~nm}$ case, Figure 14 shows that at probe powers close to the optimum power the performance of the 1542 and $1550 \mathrm{~nm}$ wavelengths is very similar, which is also reflected in Figure 14. In Figure 15 the performance of all three wavelengths are evenly spaced. Therefore, it can be concluded that the differences in performance at wavelengths in the ONU transmitter CWDM band can be controlled through the choice of probe power.

The results of the wavelength drift in the distribution section against received power were similar for both backhaul wavelengths. Figure 14 and Figure 15 show that the target performance of BER $=10^{-10}$ was achieved across the $1520.25 \mathrm{~nm}$ to $1535.04 \mathrm{~nm}$ wavelength band chosen for the DWDM upstream backhaul wavelengths.

\section{CONCLUSIONS}

In this study we have demonstrated experimentally a range of architectures that can consolidate a number of PONs with unstable wavelengths into a long-haul WDM backhaul using all optical techniques. It has been shown that although attractive from an integration perspective, the use of two SOA in the wavelength conversion stage has limitations due to the inherent gain slope of the devices. This limits the split size of the consolidated PONs to 32 users. Application of the EDFA shows an improvement in the potential split to at least 64 ports. With the addition of amplification in the backhaul section,

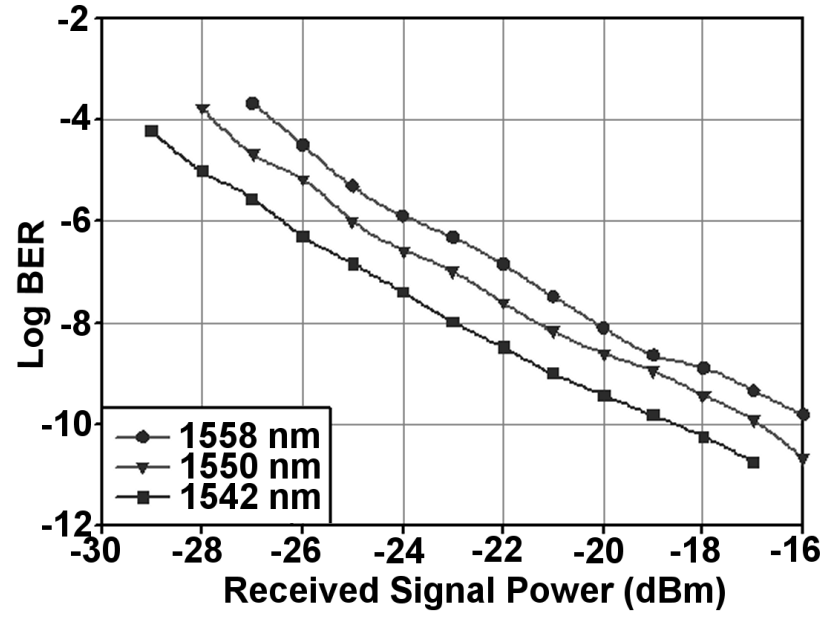

Fig. 15. System performance when converting the distribution section wavelengths to a backhaul wavelength of $1520.25 \mathrm{~nm}$.

the system can support split sizes of 128 which demonstrates that potentially 2560 ONUs could be supported by a single backhaul fiber with low-cost, uncooled laser sources at the customer premises.

\section{REFERENCES}

[1] K. Grobe, J.-P Elbers, "PON in adolescence: from TDMA to WDMPON" IEEE Commun. Mag., vol. 46 (1), pp. 26-34, 2008. 1.

[2] D. P. Shea and J. E. Mitchell "Long Reach Optical Access Technologies" IEEE Network, Sep/Oct 2007

[3] J. R. Stern, C. E. Hoppitt, D. B. Payne, M. H. Reeve, and K. Oakley, "TPON-a passive optical network for telephony," in Proc ECOC 88. 14th European Conf. on Optical Comm., 1988.

[4] "ITU-T Recommendation G.983.1: 'Broadband optical access systems based on Passive Optical Networks (PON)',' ITU 1998.

[5] IEEE Std 802.3-2005 IEEE Standard for Information technology Telecommunications and information exchange between systems-Local and metropolitan area networks -Specific requirements.

[6] "ITU-T Recommendation G.984.1: 'Gigabit-capable Passive Optical Networks (GPON): General characteristics'," ITU 2003.

[7] I. Van de Voorde, C. M. Martin, I. Vandewege, and X. Z. Oiu, "The superPON demonstrator: an exploration of possible evolution paths for optical access networks," IEEE Commun. Mag., vol. 38, pp. 74, 2000.

[8] D. P. Shea and J. E. Mitchell, "A 10 Gb/s 1024-Way Split 100-km Long Reach Optical Access Network," J. Lightwave Technol., vol 25, Issue 3, March 2007.

[9] K. Inoue, "Polarization-insensitive wavelength conversion using fibre fourwave mixing with two orthogonal pumps at different frequencies," in OFC '94. San Jose, CA: ThQ5, 1994.

[10] M. C. Tatham, G. Sherlock, and L. D. Westbrook, "20-nm optical wavelength conversion using non-degenerate four-wave mixing," IEEE Photonics Technol. Lett, vol. 5, pp. 1303, 1993.

[11] T. Durhuus, B. Mikkelsen, C. Joergensen, S. Lykke Danielsen, and K. E. Stubkjaer, "All-optical wavelength conversion by semiconductor optical amplifiers," J. Lightwave Technol., vol. 14, pp. 942, 1996.

[12] K. Inoue, T. Mukai, and T. Saitoh, "Gain Saturation Dependance on Signal Wavelength in a Travelling-Wave Semiconductor Laser Amplifier," Electron Lett., vol. 23, pp. 328 - 329, 1987.

[13] F. Koyama and K. Iga, "Frequency Chirping in External Modulators," J. Lightwave Technol., vol. 6, pp. 87 - 93, 1988.

[14] I. P. Kaminow and T. L. Koch, Optical Fiber Telecommunications IIIA, Academic Press, 1997, pp. $122-123$.

[15] D. Nesset, R. P. Davey, D. P. Shea, P. Kirkpatrick, S. Q. Shang, M. Lobel, and B. Christensen, "10 Gbit/s Bidirectional Transmission in 1024way Split, $110 \mathrm{~km}$ Reach PON Systems using Commercial Transceiver Modules, SuperFEC and EDC," in proc ECOC 2005, Glasgow, Scotland, 2005.

[16] G. P. Agrawal and N. A. Olsson, "Amplification and compression of weak picosecond optical pulses by using semiconductor-laser amplifier," Opt. Lett, vol. 14, pp. 500-502, 1989. 
[17] P. V. Mamyshev, "All-optical data regeneration based on self-phase modulation effect," in ECOC 1998., vol. 1, 1998, pp. 475.

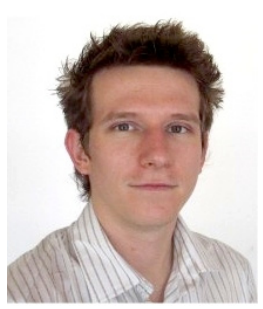

Darren P. Shea Darren Shea received his Engineering Doctorate (EngD) Degree in Telecommunications from University College London (UCL) in 2006. His thesis research, performed in conjunction with $\mathrm{BT}$, involved the design and construction of a $10 \mathrm{Gbit} / \mathrm{s}$ optical access network which connected 1024 customers directly to the core network over a distance of $100 \mathrm{~km}$. Prior to his doctorate studies, he received first class honours in electronic engineering from The University of Manchester.

For the duration of his doctorate research, Darren worked as part of the Optical Networks group at BT, based at Adastral Park, Ipswich, UK. Currently, he is working as a Research Fellow at UCL. His research interests include optical access, specifically fibre to the home, and burst mode receiver and long haul transmission improvements using optical frequency chirping.

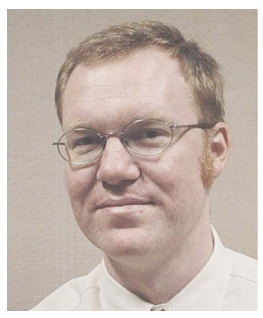

John E. Mitchell John E. Mitchell (S96 M00) received the Ph.D. degree in Electronic Engineering from UCL (University College London), UK in 2000. Since 2000 he has been a a Lecturer and Senior Lecturer (since 2006) in the Department of Electronic and Electrical Engineering at UCL.

$\mathrm{He}$ has published over 40 papers in the area of optical communications. His research interests include optical access technologies and the transport of millimetre wave radio signals over optical fibre for communications, and noise relating to crosstalk in optical networks. Dr Mitchell is a member of the Institute of Engineering and Technology (IET) and the IEEE ComSoc 